\title{
Electronic Transport Through a Quantum Wire with a Side-Coupled Quantum Dot
}

\author{
T. Lobo*, M. S. Figueira*, and M. E. Foglio ${ }^{\dagger}$ \\ *Departamento de Física, Universidade Federal Fluminense Av. Litorânea s/n, 24210-340 Niterói-RJ, Brazil \\ ${ }^{\dagger}$ Instituto de Física Gleb Wataghin, Universidade Estadual de Campinas, C. P. 6165, 13083-970 Campinas-SP, Brazil
}

Received on 4 April, 2005

\begin{abstract}
We describe the Kondo resonance in quantum dots employing the atomic model. We calculate approximate Green's functions of the impurity Anderson model employing the exact solution of the system with a conduction band with zero width, and we use the completeness condition to choose the position of that band. At low temperatures, there are two solutions close to the chemical potential $\mu$, satisfying this condition, and we choose the one with minimum Helmholtz free energy, considering that this corresponds to the Kondo solution. At high temperatures, this solution no longer exist, corresponding to the disappearance of the Kondo peak. We present curves of density of states that characterize the Kondo peak structure problem. As a simple application we calculate the conductance of a side-coupled quantum dot and we obtain good agreement with recent experimental results.
\end{abstract}

Keywords: Electronic transport; Side-coupled quantum dot; Kondo resonance

\section{CONDUCTANCE OF A SIDE-COUPLED QUANTUM DOT}

The Kondo effect explains the increased resistivity shown by a metal with magnetic impurities at low temperatures. The Kondo effect in quantum dots has been theoretically predicted since 1988 [1], and recent experiments confirmed its presence [2]. These systems can be modelled by the Anderson impurity model, and in this paper we apply the impurity atomic model to study the electronic transport through a quantum wire with a side-coupled quantum dot (QD). This system has been studied, from the theoretical point of view, [3, 4] and has been recently studied experimentally $[5,6]$.

In Fig. 1 we present a pictorial view of a simple onedimensional quantum wire with a side-coupled Anderson impurity with infinite Coulomb repulsion $U$ at site 0. The Hamiltonian of the system can be written as

$$
\begin{aligned}
H= & \sum_{\mathbf{k}, \sigma} E_{\mathbf{k}, \sigma} c_{\mathbf{k}, \sigma}^{\dagger} c_{\mathbf{k}, \sigma}+\sum_{\sigma} E_{f, \sigma} X_{f, \sigma \sigma} \\
& +V \sum_{\mathbf{k}, \sigma}\left(X_{f, 0 \sigma}^{\dagger} c_{\mathbf{k}, \sigma}+c_{\mathbf{k}, \sigma}^{\dagger} X_{f, 0 \sigma}\right),
\end{aligned}
$$

where the first term represents the noninteracting wire, characterized by free conduction electrons ( $c$-electrons), the second describes the QD described by a localized $f$ level $E_{f, \sigma}$, (we employ the $\mathrm{f}$ letter to indicate localized electrons at the impurity site) and the last one corresponds to the tunneling, where the hybridization $V$ denotes the tunneling matrix element between the QD level and the site 0 of the quantum wire, and for simplicity we consider a constant hybridization $V$. We employ the Hubbard operators [7, 8] to project out the double occupation state $|f, 2\rangle$, from the local states on the impurity. As the $X$ Hubbard operators do not satisfy the usual fermion commutation relations, the diagrammatic methods based on Wick's theorem are not applicable, and one has to use the product rules [8] $X_{f, a b} . X_{f, c d}=\delta_{b, c} X_{f, a d}$, to derive a cumulant expansion. The identity decomposition in the reduced space of local states at the impurity is given by

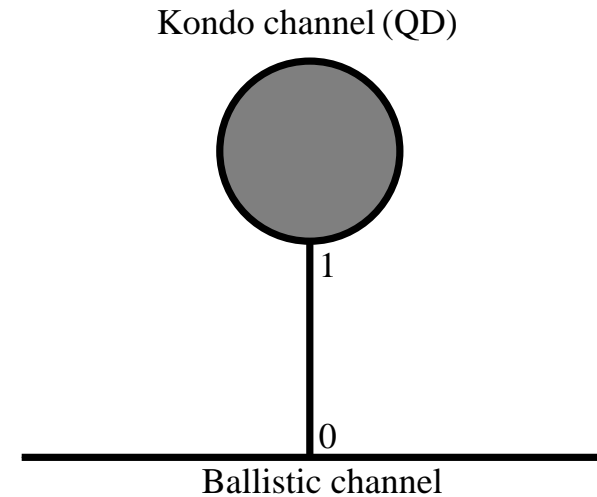

FIG. 1: Pictorial view of the quantum wire with a side-coupled quantum dot. The quantum wire is considered to be an impurity free one dimensional metal and the quantum dot is modelled by an Anderson impurity.

$$
X_{f, 00}+X_{f, \sigma \sigma}+X_{f, \overline{\sigma \sigma}}=I,
$$

where $\bar{\sigma}=-\sigma$, and the three $X_{f, a a}$ are the projectors into the states $|f, a\rangle$. The occupation numbers on the impurity $n_{f, a}=<X_{f, a a}>$ should then satisfy the "completeness" relation

$$
n_{f, 0}+n_{f, \sigma}+n_{f, \bar{\sigma}}=1 \text {. }
$$

At low temperature and bias voltage electron transport is coherent, and a linear-response conductance is given by the Landauer-type formula [3]

$$
G=\frac{2 e^{2}}{\hbar} \int\left(-\frac{\partial n_{f}}{\partial \omega}\right) S(\omega) d \omega
$$

where $n_{f}$ is the Fermi function and $S(\omega)$ is the transmission probability of an electron with energy $\hbar \omega$. This probability is 
given by

$$
S(\omega)=\Gamma^{2}\left|G_{00}^{\sigma}\right|^{2},
$$

where $\Gamma$ corresponds to the coupling strength of the site 0 to the wire (which is proportional to the kinetic energy of the electrons in the wire). $G_{00}^{\sigma}$ can be calculated by the Dyson equation with $\tilde{V}=|0\rangle V\langle 1|+| 1\rangle V\langle 0|$ being the hybridization. The dressed Green's functions at the site 0 can be written in terms of the undressed Green's functions localized at the QD, $g_{11}$, and the undressed Green's functions of the conduction electrons, $g_{00}$

$$
\begin{aligned}
& G_{00}^{\sigma}=g_{00}^{\sigma}+g_{00}^{\sigma} V G_{10}^{\sigma}+g_{01}^{\sigma} V G_{00}^{\sigma}, \\
& G_{10}^{\sigma}=g_{10}^{\sigma}+g_{10}^{\sigma} V G_{10}^{\sigma}+g_{11}^{\sigma} V G_{00}^{\sigma} .
\end{aligned}
$$

Solving this system of equations, and considering $g_{10}=0$ and $g_{01}=0$, we can write

$$
G_{00}^{\sigma}=\frac{g_{00}^{\sigma}}{\left(1-g_{00}^{\sigma} V^{2} g_{11}^{\sigma}\right)},
$$

where

$$
g_{00}^{\sigma}=\left(\frac{-1}{2 D}\right) \ln \left(\frac{z+D+\mu}{z-D+\mu}\right) \quad ; \quad g_{11}^{\sigma}=M_{2, \sigma}^{a t}(z) .
$$

The $g_{00}$ is the free Green function of the quantum ballistic channel, with $\mu$ being the chemical potential and $M_{2, \sigma}^{a t}(z)$ the approximate cumulant of the QD, obtained through the atomic model calculated in Sec. II.

\section{THE ATOMIC MODEL}

To obtain the exact $f$ Green function $G_{f f, \sigma}\left(\mathbf{j}_{i}, z\right)$ in real space for the impurity at site $\mathbf{j}_{i}$, one can follow a procedure similar to the one used in [9] within the chain approximation, but considering all the possible cumulants in the expansion as it was done in [10] for the Anderson lattice. As with the Feynmann diagrams, one can rearrange all those that contribute to the exact $G_{f f, \sigma}\left(\mathbf{j}_{i}, z\right)$ by defining an effective cumulant $M_{2 \sigma \sigma}^{e f f}\left(\mathbf{j}_{i}, z\right)$, that is given by all the diagrams of $G_{f f, \sigma}\left(\mathbf{j}_{i}, z\right)$ that can not be separated by cutting a single edge (usually called "proper" or "irreducible" diagrams). We shall consider that the impurity is at the origin, and drop the index $\mathbf{j}_{i}$ from all the quantities. The exact $\mathrm{GF} G_{f f, \sigma}(z)$ is then given by replacing the bare cumulant $M_{2, \sigma}^{0}(z)=-D_{\sigma}^{0} /\left(z-\varepsilon_{f}\right)$ by the effective cumulant $M_{2, \sigma}^{e f f}(z)$ at all the filled vertices of the chain diagrams in [9]. The exact GF for the f electron is then written as

$$
G_{f f, \sigma}(z)=\frac{M_{2, \sigma}^{e f f}(z)}{1-M_{2, \sigma}^{e f f}(z)|V|^{2} \sum_{\mathbf{k}} G_{c, \sigma}^{o}(\mathbf{k}, z)},
$$

where $G_{c, \sigma}^{o}(\mathbf{k}, z)=-1 /(z-\varepsilon(\mathbf{k}))$. The exact GF in the atomic limit $G_{f f, \sigma}^{a t}(z)$ has the same form of Eq. (10 and can be calculated exactly. It is given in the Appendix (cf. Eq. 15):

$$
G_{f f, \sigma}^{a t}(z)=\frac{M_{2, \sigma}^{a t}(z)}{1-M_{2, \sigma}^{a t}(z)|V|^{2} \sum_{\mathbf{k}} G_{c, \sigma}^{o}(\mathbf{k}, z)} .
$$

From this equation we then obtain an explicit expression for $M_{2, \sigma}^{a t}(z)$ in terms of $G_{f f, \sigma}^{a t}(z)$

$$
M_{2, \sigma}^{a t}(z)=\frac{G_{f f, \sigma}^{a t}(z)}{1+G_{f f, \sigma}^{a t}(z)|V|^{2} \sum_{\mathbf{k}} G_{c, \sigma}^{o}(\mathbf{k}, z)} .
$$

To decrease the contribution of the $c$ electrons, whose effect was overestimated by concentrating them at a single energy level we shall replace $V^{2}$ by $\Delta^{2}$, where $\Delta=\pi V^{2} / 2 D$ is of the order of the Kondo peak's width. The atomic approximation consists in substituting $M_{2, \sigma}^{e f f}(z)$ in Eq. (10) by the approximate $M_{2, \sigma}^{a t}(z)$ given by Eq. (12). As $M_{2, \sigma}^{a t}(z)$ is $\mathbf{k}$ independent, we can easily obtain the exact local Green function for the Anderson impurity for a square band of bandwidth $2 D$

$$
G_{f f, \sigma}^{i m p}(z)=\frac{M_{2, \sigma}^{a t}(z)}{1+M_{2, \sigma}^{a t}(z) \frac{|V|^{2}}{2 D} \ln \left(\frac{z+D+\mu}{z-D+\mu}\right)},
$$

and in the same way we obtain the conduction $\left(G_{c}\right)$ and mixed $\left(G_{f c}\right)$ Green's functions. The difference between the exact and the approximate quantity is that different energies $E_{\mathbf{k}, \sigma}$ appear in the c-electron propagators of the effective cumulant $M_{2, \sigma}^{e f f}(z)$, while these energies are all equal to $E_{0}$ in $M_{2, \sigma}^{a t}(z)$. Although $M_{2, \sigma}^{a t}(z)$ is only an approximation, it contains all the diagrams that should be present, and one would expect that the corresponding GF would have fairly realistic features. One still has to decide what value of $E_{0}=E_{q}$ should be taken. As the most important region of the conduction electrons is the Fermi energy, we shall use $E_{0}=\mu-\delta E_{0}$, leaving the freedom of small changes $\delta E_{0}$ to adjust the results in such way that the completeness relation given by Eq. (2) should be satisfied.

\section{RESULTS AND CONCLUSIONS}

In Fig. 2 we represent the completeness $n_{o}+2 n_{f}$ as function of the position of the atomic conduction band $E_{q}$. We can see that at very low temperatures, there are two solutions close to the chemical potential $\mu$, satisfying this condition, and we choose the one with minimum Helmholtz free energy, so we consider that this corresponds to the Kondo solution. In the same figure we can see that if we increase the temperature this solution disappears, and this corresponds to the vanishing of the Kondo peak in the density of states.

In Fig. 3 we plot the density of states corresponding to a Kondo situation. We can see the two structures characteristic of Kondo densities of states: one non-resonant peak located in the $E_{f}^{o}$ position and the Kondo peak located on the chemical potential $\mu$. In the inset we represent a detail of the Kondo peak.

In Fig. 4 we represent the conductance of a side-coupled quantum dot. In this geometry there is an anti-Kondo resonance at very low temperatures, in the Kondo region. The Kondo effect at the dot produce a destructive interference between the electrons that follow the ballistic channel and the electrons that go to the dot. The result is that in the Kondo region and at low temperatures the conductance $G / G_{o}$, goes 


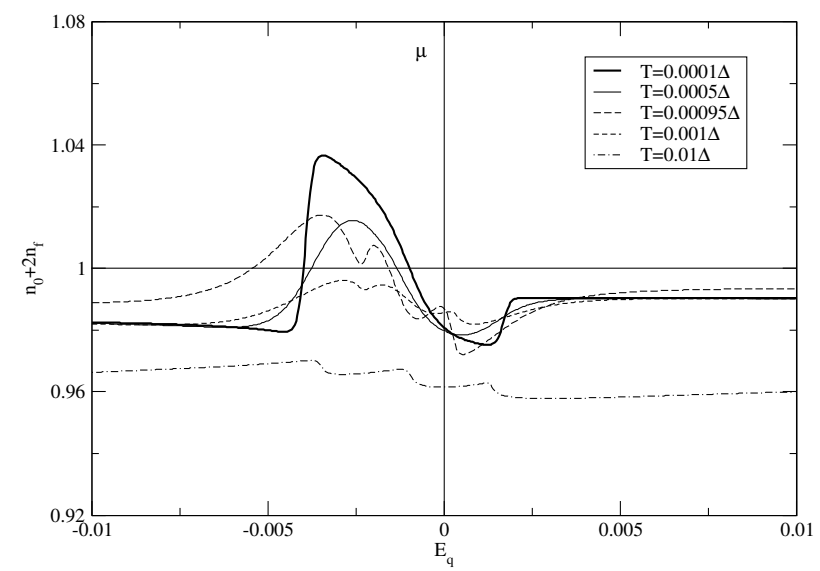

FIG. 2: Completeness as function of $E_{q}$ for different temperatures.

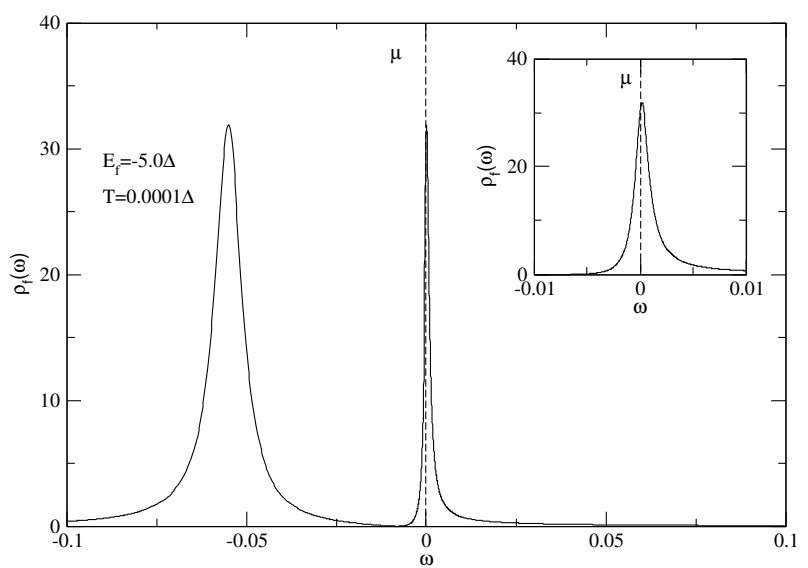

FIG. 3: Density of states for $T=0.0001 \Delta$ with $E_{f}=-5.0$. Detail of the Kondo peak formation.

to zero as we can see in the Fig. 4. This result agrees well with recent experimental results [5].

\section{Apendix: Atomic solution}

We assume a zero width conduction band $2 D=0$, so that the hoping contributions are eliminated from the Hamiltonian. In this case we have a Fock space with twelve states characterized by $\mid m, \sigma>$ as can be seen in table 1

There is now an independent system at each site, with the Anderson impurity at one of them, and the system Hamiltonian can be diagonalized exactly. There are four possible states at each normal site: $(0, \uparrow, \downarrow, \uparrow \downarrow)$, while there are only three $m$ extra states $\left(0,+\frac{1}{2},-\frac{1}{2}\right)$ at the impurity site ( without a doubly occupied state because $U \rightarrow \infty$ ). At the impurity we then have a Fock space with the twelve states $|m, \sigma\rangle$ described in Table 1. To obtain the atomic Greens functions we

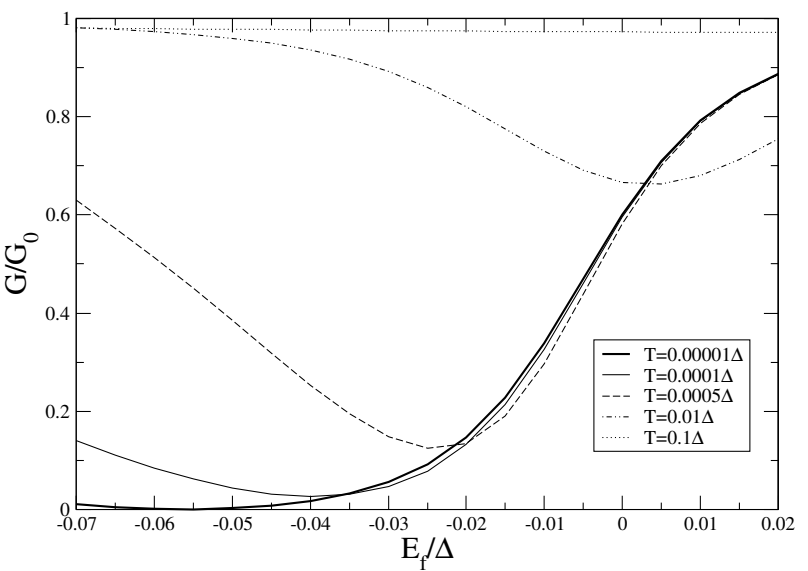

FIG. 4: Conductance of a side-coupled quantum dot.

TABLE I: States of the Anderson lattice in the limit $2 D=V=0$.

\begin{tabular}{|l|c|c|c|}
\hline $\mid m, \sigma>$ & $\mathrm{E}$ & $\mathrm{n}$ & $S_{z}$ \\
\hline $\mid 0,0>$ & 0 & 0 & 0 \\
\hline $\mid \frac{1}{2}, 0>$ & $\varepsilon_{f}$ & 1 & $1 / 2$ \\
\hline $\mid-\frac{1}{2}, 0>$ & $\varepsilon_{f}$ & 1 & $-1 / 2$ \\
\hline $\mid 0, \uparrow>$ & $\varepsilon_{q}$ & 1 & $1 / 2$ \\
\hline $\mid 0, \downarrow>$ & $\varepsilon_{q}$ & 1 & $-1 / 2$ \\
\hline $\mid \frac{1}{2}, \uparrow>$ & $\varepsilon_{f}+\varepsilon_{q}$ & 2 & 1 \\
\hline $\mid \frac{1}{2}, \downarrow>$ & $\varepsilon_{f}+\varepsilon_{q}$ & 2 & 0 \\
\hline $\mid-\frac{1}{2}, \uparrow>$ & $\varepsilon_{f}+\varepsilon_{q}$ & 2 & 0 \\
\hline $\mid-\frac{1}{2}, \downarrow>$ & $\varepsilon_{f}+\varepsilon_{q}$ & 2 & -1 \\
\hline $\mid 0, \uparrow \downarrow>$ & $2 \varepsilon_{q}$ & 2 & 0 \\
\hline $\mid \frac{1}{2}, \uparrow \downarrow>$ & $\varepsilon_{f}+2 \varepsilon_{q}$ & 3 & $1 / 2$ \\
\hline $\mid-\frac{1}{2}, \uparrow \downarrow>$ & $\varepsilon_{f}+2 \varepsilon_{q}$ & 3 & $-1 / 2$ \\
\hline
\end{tabular}

TABLE II: Energies of the twelve states $|n, r\rangle$ of $\mathcal{H}$. The columns give the number of electrons $n$ in the state $r$, the spin component $S_{z}$ and $\varepsilon_{n, r}=E_{n, r}-n \mu$, where $E_{n, r}$ is the energy of the state $|n, r\rangle$, $\Delta=\sqrt{\left(E_{0}-E_{f}\right)^{2}+4 V^{2}}$ and $\Delta^{\prime}=\sqrt{\left(E_{0}-E_{f}\right)^{2}+8 V^{2}}$.

\begin{tabular}{|l|l|l|l|}
\hline$n$ & $r$ & $S_{z}$ & $\varepsilon_{n, r}=E_{n, r}-n \mu$ \\
\hline 0 & 1 & 0 & $\varepsilon_{q}$ \\
\hline 1 & 2 & $+1 / 2$ & $1 / 2\left(\varepsilon_{f}+\varepsilon_{q}-\Delta\right)$ \\
\hline 1 & 3 & $-1 / 2$ & $1 / 2\left(\varepsilon_{f}+\varepsilon_{q}-\Delta\right)$ \\
\hline 1 & 4 & $+1 / 2$ & $1 / 2\left(\varepsilon_{f}+\varepsilon_{q}+\Delta\right)$ \\
\hline 1 & 5 & $-1 / 2$ & $1 / 2\left(\varepsilon_{f}+\varepsilon_{q}+\Delta\right)$ \\
\hline 2 & 6 & +1 & $\varepsilon_{f}+\varepsilon_{q}$ \\
\hline 2 & 7 & -1 & $\varepsilon_{f}+\varepsilon_{q}$ \\
\hline 2 & 8 & 0 & $\varepsilon_{f}+\varepsilon_{q}$ \\
\hline 2 & 9 & 0 & $1 / 2\left(\varepsilon_{f}+3 \varepsilon_{q}-\Delta^{\prime}\right)$ \\
\hline 2 & 10 & 0 & $1 / 2\left(\varepsilon_{f}+3 \varepsilon_{q}+\Delta^{\prime}\right)$ \\
\hline 3 & 11 & $+1 / 2$ & $\varepsilon_{f}+2 \varepsilon_{q}$ \\
\hline 3 & 12 & $-1 / 2$ & $\varepsilon_{f}+2 \varepsilon_{q}$ \\
\hline
\end{tabular}


use the Zubarev's equation

$$
\begin{aligned}
G_{\omega}^{a t}(\omega)= & e^{\beta \Omega} \sum_{n} \sum_{j j^{\prime}}\left(e^{-\beta E_{n j}}+e^{-\beta E_{n-1 j^{\prime}}}\right) \times \\
& \frac{\left|<n-1, j^{\prime}\right| X_{\mu}|n j>|^{2}}{\omega-\left(E_{n j}-E_{n-1 j^{\prime}}\right)}
\end{aligned}
$$

where $\Omega$ is the thermodynamical potential and the eigenvalues $E_{n j}$ and eigenvectors $\mid n j>$ corresponds to the complete solution of the Hamiltonian. The final result is the following

$$
G^{a t}(\omega)=e^{\beta \Omega} \sum_{i=1}^{8} \frac{m_{i}}{\omega-u_{i}},
$$

where the poles of the Green's functions are given by

$$
\begin{gathered}
u_{1}=E_{3}-E_{1}=E_{8}-E_{5}=E_{7}-E_{4}=\frac{1}{2}\left(\varepsilon_{f q}-\Delta\right) ; \\
u_{2}=E_{5}-E_{1}=E_{8}-E_{3}=E_{7}-E_{2}=\frac{1}{2}\left(\varepsilon_{f q}+\Delta\right) ; \\
u_{3}=E_{12}-E_{10}=\frac{1}{2}\left(\varepsilon_{f q}-\Delta^{\prime}\right) ; \\
u_{4}=E_{12}-E_{9}=\frac{1}{2}\left(\varepsilon_{f q}+\Delta^{\prime}\right) ; \\
u_{5}=E_{9}-E_{2}=\varepsilon_{q}-\frac{1}{2}\left(\Delta^{\prime}-\Delta\right) ; \\
u_{6}=E_{10}-E_{2}=\varepsilon_{q}+\frac{1}{2}\left(\Delta^{\prime}+\Delta\right) ; \\
u_{7}=E_{9}-E_{4}=\varepsilon_{q}-\frac{1}{2}\left(\Delta^{\prime}+\Delta\right) ; \\
u_{8}=E_{10}-E_{4}=\varepsilon_{q}+\frac{1}{2}\left(\Delta^{\prime}-\Delta\right) .
\end{gathered}
$$

and the residues are easily calculated employing Eq.(14) with the exact eigenvectors $\mid n j>$ of the atomic Hamiltonian. We have used $\varepsilon_{f q}=\varepsilon_{f}+\varepsilon_{q}$.
[1] L. I. Glazman and M. E. Raikh JETP Lett. 47, 452 (1988).

[2] D. Goldhaber-Gordon et al., Nature 391, 156 (1998).

[3] K. C. Kang, S. Y. Cho, J. J. Kim, and S. C. Shin, Phys. Rev. B 63, 113304 (2001)

[4] R. Franco, M. S. Figueira, and E. V. Anda, Phys. Rev. B 67, 155301 (2003).

[5] Masahiro Sato, Hisashi Aikawa, Kensuke Kobayashi, Shingo Katsumoto, and Yashiro Iye, Phys. Rev. Letters 95, 066801 (2005).
[6] K. Kobayashi, H. Aikawa, A. Sano, S. Katsumoto, and Y. Iye, Phys. Rev. B, 70, 035319 (2004).

[7] J. Hubbard, Proc. R. Soc. London, Ser. A 285, 542 (1965).

[8] M. S. Figueira, M. E. Foglio, and G. G. Martinez, Phys. Rev. B 50, 17933 (1994).

[9] R. Franco, M. S. Figueira, and M. E. Foglio, Phys. Rev. B 66, 45112 (2002).

[10] M. E. Foglio and M. S. Figueira, Phys. Rev. B 60, 11361 (1999). 